\title{
Quality-Assured Energy Balancing for Multi-hop Wireless Multimedia Networks via 2-D Channel Coding Rate Allocation
}

\author{
Lin Xing, Wei Wang, Gensheng Zhang \\ Electrical Engineering and Computer Science, \\ South Dakota State University, USA \\ Ixing@jacks.sdstate.edu, \\ wei.wang@sdstate.edu, \\ gensheng.zhang@jacks.sdstate.edu
}

\author{
Feifei Gao, Xuewen Liao, Tigang Jiang \\ Tsinghua University, China; Xian Jiaotong \\ University, China; University of Electronic, \\ Science and Technology of China \\ feifeigao@mail.tsinghua.edu.cn, \\ xwliao@mailst.xjtu.edu.cn, \\ jtg@uestc.edu.cn
}

\begin{abstract}
How to prolong the multimedia application based on multihop wireless mesh networks powered by finite energy resource is a critical issue. In this paper, we propose a new approach to extend network lifetime by maximizing energybalancing between routers while satisfying minimal multimedia service quality requirement. Specifically, a new channel coding scheduling algorithm is proposed to allocate the optimal channel coding redundancy to each multimedia packet to achieve both the maximal energy-balancing of the network and the lower bound of multimedia Quality of Service (QoS) requirement. The simulation results show that the proposed approach can significantly improve the energybalancing of the multi-hop wireless mesh networks.
\end{abstract}

\section{Categories and Subject Descriptors}

H.4 [Information Systems Applications]: Miscellaneous; C.2.1 [Network Architecture and Design]: Cross-layer Design —wireless networks, multimedia computing

\section{General Terms \\ Design}

\section{Keywords}

Channel Coding, Image Transmission, Energy Balancing, Multi-hop Wireless Multimedia Networks

\section{INTRODUCTION}

Multimedia streaming application over multi-hop wireless mesh network has been applied to wide areas to provide low cost communication service recently. Different from wired network, routers in a mobile and embedded wireless network are usually powered by finite battery with limited energy resource. Extending the lifetime of multi-hop wireless

Permission to make digital or hard copies of all or part of this work for personal or classroom use is granted without fee provided that copies are not made or distributed for profit or commercial advantage and that copies bear this notice and the full citation on the first page. To copy otherwise, to republish, to post on servers or to redistribute to lists, requires prior specific permission and/or a fee.

RACS '11, November 2-5, 2011, Miami, FL, USA.

Copyright 2011 ACM 978-1-4503-1087-1/11/11 ...\$10.00. mesh network powered by battery is a critical design consideration, which is especially important if it is hard or impossible to recharge or replace exhausted battery for router in some extreme cases. Network lifetime in this paper is defined as the duration before the first router runs out of battery. Because once a router exhaust energy and disconnect with other routers, the function of the network will be seriously damaged. It is especially true if the router is in a crucial position of a path or is a significant pivot of the whole network. However, routers at different positions may have different energy-consumption because they have different workloads and are under different channel conditions. The lifetime of the network will be reduced if a router always consumes more energy than others. Improving the energy-balancing of the whole wireless network can significantly extend the lifetime of the network, since it has been widely accepted that applying the same energy-allocation to all routers in the network is not an advisable strategy.

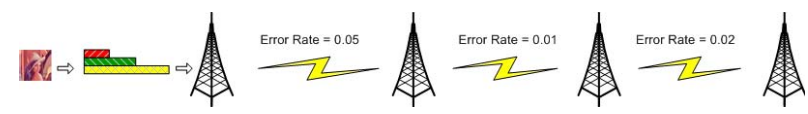

Figure 1: Illustration of optimal energy-balancing scheduling in Multi-Hop Wireless Mesh Networks

Recent research in the area of multi-hop wireless multimedia mesh network were focus on multimedia QoS provision and the optimal system scheduling with end-to-end delay constraint, which seldom considered prolonging the lifetime of wireless mesh network. In research [1], the authors surveyed the scheduling technique for wireless networks, in which several scheduling algorithms were studied for TDMA, CDMA, and multi-hop packet networks. In research [2], the authors proposed a collaborative algorithm considering quality of service requirements, the underlying channel conditions, and the network topology to maximize Point to Point (P2P) multimedia quality through multi-hop wireless networks. The proposed algorithm optimizes resource exchanges by cooperatively allocate available resource among peers. In addition, scalable coding and cross-layer strategies are used to enable the resource exchanges. The advantage of multiple antennas was exploited in research [3] to support multimedia application in multi-hop wireless networks. In research [4], the authors solved the problem that how to route multiple de- 
scription multimedia video in multi-hop wireless networks by designing cross-layer optimal strategy with an application performance metric. A branch-and-bound solution and a new technique called Reformulation-Linearization Technique (RLT) were exploited to find the optimal solution. In research [5], the authors proposed an integrated cross-layer algorithm which optimized various control parameters across the protocol layers and across the various nodes to maximize the video quality in multi-hop wireless mesh networks with delay-constraint. The algorithm was performed by various scenarios with different levels of network status and the results showed a significant improvement of video QoS. In research [6], the authors investigated improvement of unreliability of video streaming in wireless multi-hop networks under a cross-layer optimization framework which considered various cross-layer parameters at the physical layer, medium access control (MAC) layer, and application layer to maximize the video quality, where optimal deployment of path diversity was also studied to solve the link error problem. A distributed scheduling was developed in research [7] to achieve minimal multimedia-distortion and fairness by testing media distortion and allocating network resource. In research [8], the authors deployed an overlay infrastructure to feedback necessary network conditions for cross-layer adaptation. Some other cross-layer design about MAC, routing, energy distribution, and rate allocation were studied in [9] [10] to achieve the optimal overall system objective. However, the primary purpose of studies above was to maximize the decoded user-received multimedia quality, which did not consider the energy constraint - a critical factor for batterypowered wireless mesh networks.

\section{PROBLEM STATEMENT}

In this paper, we focus on the energy-balancing along a single communication path and multimedia packets have various quality contribution. A scenario of the multi-hop wireless mesh network discussed in this paper is illustrated in Figure 1. As Figure 1 shows, a multimedia file that contains some multimedia packets with different size and different importance level waits to be transmitted from the source node to the sink node along a single communication path. Each multimedia packet need to pass all routers in the liner transmission chain, which means multimedia packet can only be transmitted between two adjacent routers. Routers along the path have different residual energy and are under various channel conditions. After transmitting the multimedia file, the energy-balancing of the network should be optimal and end-to-end quality satisfy the minimal quality requirement.

In a linear chain topology of multi-hop wireless mesh network, once a router runs out of the battery, the whole transmission path will be cut off leading to loss of communication support, even other routers may still have much residual energy. In this paper, we extend the lifetime of wireless network by improving the energy-balancing of whole network, the energy-balancing is defined as the difference between two nodes with the highest and the lowest residue energy respectively in [11], and we follow the symbol definition presented in [12]. Thus the energy-balancing optimization could be formulated as:

$$
[G]=\arg \min \left\{\max _{\forall i \in S}\left(e_{i}\right)-\min _{\forall j \in S}\left(e_{j}\right)\right\}
$$

Subject to the QoS lower bound constraint:

$$
E[D] \geq D_{\min }
$$

Where $[G]$ denotes the optimal channel coding rate allocation of each multimedia packet on each hop, which leads to the minimal energy-difference between the "highest energy" router and the "lowest energy" router. $S$ denotes the set of all routers along the linear chain wireless mesh network, $e_{i}$ and $e_{j}$ denotes the residue energy of router $i$ and router $j$ respectively. $\max \left(e_{i}\right)$ and $\min \left(e_{j}\right)$ are the two routers with the highest and the lowest residue energy respectively. The purpose of equation 1 is to optimize the channel rate allocation in each hop along the linear router chain to minimize the energy-difference between routers in the network. $E[D]$ in equation 2 denotes the expected end-received multimedia quality and $D_{\min }$ is the quality-constraint that define the minimum QoS requirement. The proposed optimal quality-assured energy-balancing scheduling in this paper integrates both equation 1 and equation 2 to minimize the energy-difference between routers in the network by optimizing channel rate allocation to each hop while satisfying the transmission quality constraint.

\section{MULTIMEDIA QUALITY MODELING}

Fundamentally different from traditional approach which always apply maximal channel coding rate to all hops to achieve the maximal end-received quality, we propose a new optimal energy-balancing scheduling which allocate the optimal channel coding rate to each hop in the process of multimedia data transmission to improve the energy-balancing of the whole network, so as to prolong the lifetime of wireless mesh network.

First we analyze the expected multimedia quality received in the destination by considering the quality contribution of each packet and related path-pass probability. We have $N$ multimedia packets with various importance levels:

$$
\left\{d_{1}, d_{2}, \ldots \ldots, d_{N}\right\}
$$

These packets need to be transmitted over wireless network from the source node to the sink node, and we also have $H$ hops:

$$
\left\{h_{1}, h_{2}, \ldots \ldots, h_{H}\right\}
$$

In the wireless multimedia network, the expected end-to-end multimedia quality can be expressed as follows:

$$
E[D]=\sum_{i=1}^{C}\left(d_{i} \times \prod_{\alpha=1}^{H}\left(1-P_{i \alpha}\right)\right)
$$

$C$ denotes the number of multimedia packets transmitted over the network, which is specified after grouping multimedia packet according to importance level. $d$ denotes the expected quality contribution of a certain multimedia packet after the packet passes through the transmission path hopby-hop and received by the sink node in the destination correctly. $P_{i \alpha}$ denotes the link-loss probability of packet $i$ on 
hop $\alpha$, which means the loss probability of packet $i$ between router $\alpha$ and router $\alpha+1$. As the equation shows, the expected end-received multimedia quality is expressed as the quality-contribution summation of all packets weighted with related packet path-pass probability, which is presented as a consecutive multiplication of link-pass probability.

\section{CHANNEL CODING RATE ASSIGNMENT}

Our optimal energy-balancing scheduling is deployed with quality-constraint, so the link-layer packet loss probability is required to estimate the end-received multimedia quality. Due to the error resilience of Forward Error Coding (FEC), packet loss probability can be reduced after channel coding redundancy is added into original multimedia packet. In general, with the same original packet size and under the same channel condition, more channel coding redundancy means more packet transmission reliability and more energy consumption. The packet link loss probability that influenced by original multimedia packet size, channel coding redundancy, and channel bit loss probability can be expressed as follows according to [13]:

$$
p=\sum_{i=M+1}^{M+a}\left(\begin{array}{l}
M+a \\
i
\end{array}\right) b^{i}(1-b)^{M+a-i}
$$

where $p$ denotes the packet link loss probability, $M$ denotes the length of original multimedia packet, $a$ is the length of channel coding redundancy added into original multimedia packet, and $b$ denotes the bit loss probability. From this equation we can see different redundancy coding added to multimedia packet can cause different packet link-loss probabilities which will influence end-received multimedia quality. The proposed scheduling applies the optimal channel coding rate strategy to each packet to tune the tradeoff between QoS and energy-balancing.

To achieve the optimal channel coding rate strategy, accurate energy-consuming estimation for each router is required. Our purpose is to get the minimum energy-difference between the "highest energy" and the "lowest energy" routers after all multimedia packets are transmitted along the network. The energy consumption of each router after all multimedia packet passed is estimated as:

$e_{\text {co }}=\sum_{i=1}^{C} P_{r} \times\left(\frac{l_{i} \times g_{i_{-i n}}}{r}+t_{o}\right)+\sum_{i=1}^{C} P_{t} \times\left(\frac{l_{i} \times g_{i_{-} \text {out }}}{r}+t_{o}\right)$

where $e_{c o}$ denotes the energy consumption of a certain router, $P_{r}$ and $P_{t}$ denote the transmission power and receiving power respectively. $l_{i}$ is the length of original multimedia packet $i$, $g_{i_{-} \text {in }}$ and $g_{i_{-} \text {out }}$ denote the channel coding rate allocated to the packet $i$ on the previous router and the current router respectively, $r$ denotes the transmission data rate in the multihop wireless mesh network and $t_{o}$ is protocol overhead. This equation estimates the total energy consumption of a certain router after receiving and sending all $C$ data packets. Then the residue energy $e$ of a certain router could be calculated as

$$
e \leftarrow e-e_{c o}
$$

The optimal energy-balancing of the multi-hop wireless mesh network can be achieved by allocating the optimal channel coding rate to each multimedia packet on each hop of the network. The design guideline of proposed quality-assured energy-balancing scheduling is described as: First, estimate the residue energy of each router and the expected endreceived quality contributed by all packets with different channel coding allocation strategies; Second, search the optimal channel coding allocation scheme which leads to the minimal energy-difference of the network while the expected end-received quality satisfies the minimal quality requirement.

\section{SIMULATION}

In this section, we perform simulation study of the proposed approach and evaluate the performance of optimizing energy-balancing of the whole network and to check whether the proposed approach can transmit more multimedia packets. Traditional approach is also evaluated for comparison, which always applies the maximum coding rate in each hop to obtain the maximum end-received quality. We focus on the energy-balancing of forwarding routers in the network, because in the proposed linear multi-hop wireless mesh network topology, the source node and the sink node only send or receive data, so both of them always consume less energy than forwarding routers.

The simulation parameters are stated as follows. The multihop wireless network model includes one source node, one sink node, and six forwarding routers in the middle. The multimedia file contains five types of multimedia packet with different size and different importance level, and there are many files waiting for transmission. Transmission and receiving power is $20 \mathrm{~mW}$ and $15 \mathrm{~mW}$ respectively. The packet header of each multimedia packet is 6 bytes. Four preselected channel coding rate is $9 / 9,10 / 9,13 / 9$, and 15/9. The transmission rates in each router are shown in Table 1 with various transmission rate difference that presented as a percent of the lowest receiving rate. To focus on the energybalancing impacted by the proposed approach, evaluations for all routers were performed under the same channel condition in both proposed approach and traditional approach.

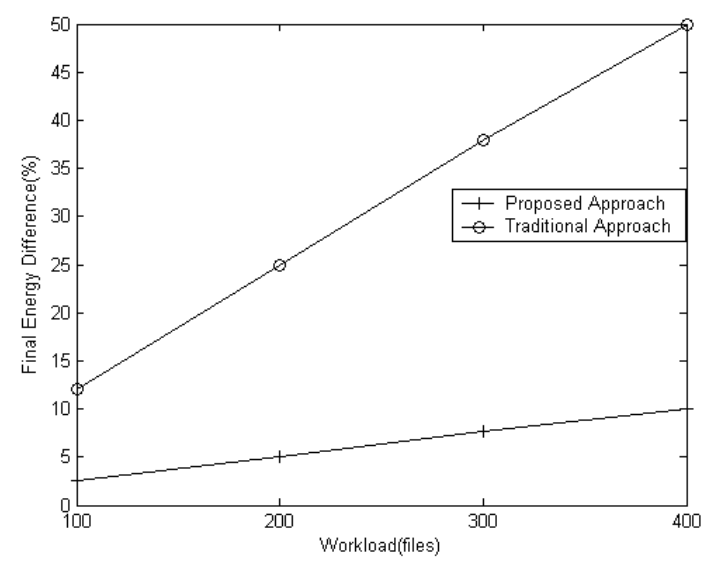

Figure 2: Maximum energy discrepancy with various workloads (transmission rate difference is $20 \%$, minimum end to end quality requirement is 356510659 MSE, bit loss provability = 3/100000)

Figure 2 illustrates the energy-difference between the "high- 
Table 1: Receiving rate and sending rate in each router with various transmission Rate Difference. receiving rate, $\mathrm{S}$ : sending rate, the unit of transmission rate is Mbps)

\begin{tabular}{|l|l|l|l|l|l|l|l|l|l|l|l|l|}
\hline \multirow{2}{*}{ TX Rate Diff. } & Router 1 & \multicolumn{2}{l|}{ Router 2} & \multicolumn{2}{l|}{ Router 3} & \multicolumn{2}{l|}{ Router 4} & \multicolumn{2}{l|}{ Router 5} & \multicolumn{2}{l|}{ Router 6} \\
\cline { 2 - 15 } & $\mathrm{R}$ & $\mathrm{S}$ & $\mathrm{R}$ & $\mathrm{S}$ & $\mathrm{R}$ & $\mathrm{S}$ & $\mathrm{R}$ & $\mathrm{S}$ & $\mathrm{R}$ & $\mathrm{S}$ & $\mathrm{R}$ & $\mathrm{S}$ \\
\hline $5 \%$ & 10 & 10.5 & 10.5 & 11 & 11 & 11.5 & 11.5 & 12 & 12 & 12.5 & 12.5 & 13 \\
\hline $10 \%$ & 10 & 11 & 11 & 12 & 12 & 13 & 13 & 14 & 14 & 15 & 15 & 16 \\
\hline $15 \%$ & 10 & 11.5 & 11.5 & 13 & 13 & 14.5 & 14.5 & 16 & 16 & 17.5 & 17.5 & 19 \\
\hline $20 \%$ & 10 & 12 & 12 & 14 & 14 & 16 & 16 & 18 & 18 & 20 & 20 & 22 \\
\hline $25 \%$ & 10 & 12.5 & 12.5 & 15 & 15 & 17.5 & 17.5 & 20 & 20 & 22.5 & 22.5 & 25 \\
\hline
\end{tabular}

est energy" router and the "lowest energy" router with different workloads that presented as the number of multimedia file transmitted. Energy-difference is presented as a percent of full charged battery. The initial energy in each router is the same but transmission rate is different, so the energy consumption in each router is different. From the figure, we can see that energy-difference increase with bigger workload, since transmission consumption in each router is different, so with more workload, more energy-difference will be. Compared with traditional approach which focuses on maximizing the end-received multimedia quality, the proposed approach can significantly improve energy-balancing of the multi-hop wireless mesh network. This is because traditional approach always applies the maximal channel coding rate to each hop without considering energy-balancing; on the other hand, the proposed scheduling can improve the energy-balancing of the network by jointly considering energy-difference, QoS, channel condition, and importance level of multimedia packet. The optimal channel coding allocation is achieved by the proposed scheduling algorithm, in which flexible channel coding rates are applied to each hop when different multimedia packet passes through. In addition, the proposed approach groups and prioritizes multimedia packets according to importance level and allocates the optimal local quality requirement to each group to make the expected global QoS satisfies the minimal quality requirement.

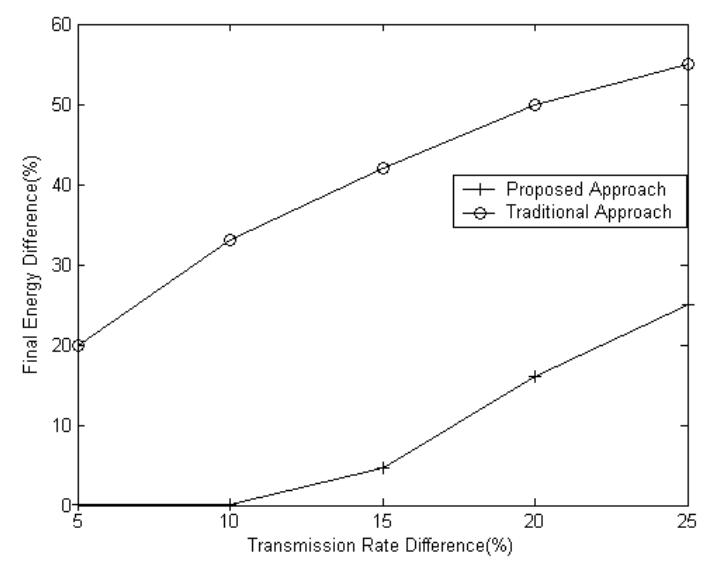

Figure 3: Maximum energy discrepancy with various transmission rate differences (minimum end-toend quality requirement is $356510659 \mathrm{MSE}$, bit loss provability $=3 / 100000$ )
Figure 3 illustrates the final energy-difference with different transmission rate difference. This experiment aims to estimate the proposed approach's ability to balance energydifference. Transmission consumption difference increased with the increase of transmission rate difference, so the energydifference also increased. As shown in the figure, the energydifference of the traditional approach increased quickly, because the traditional approach can not improve the energybalancing. However, the proposed approach almost totally balances the energy distribution between nodes in the network if the transmission rate difference is low. In our case, if the transmission rate difference less than $10 \%$, with corresponding maximum receiving rate difference less than $50 \%$ (the minimum receiving rate is $10 \mathrm{Mbps}$ and the maximum receiving rate is $15 \mathrm{Mbps}$ ). The final energy-difference of proposed approach becomes higher if transmission rate difference more than $10 \%$. That is because the energy-difference is too large to be balanced; otherwise we can extend the range of preselected channel coding rates to increase balancing capability of the proposed approach. Compared with traditional approach, the proposed approach allocates optimal resource to each router and significantly improves the energy-balancing.

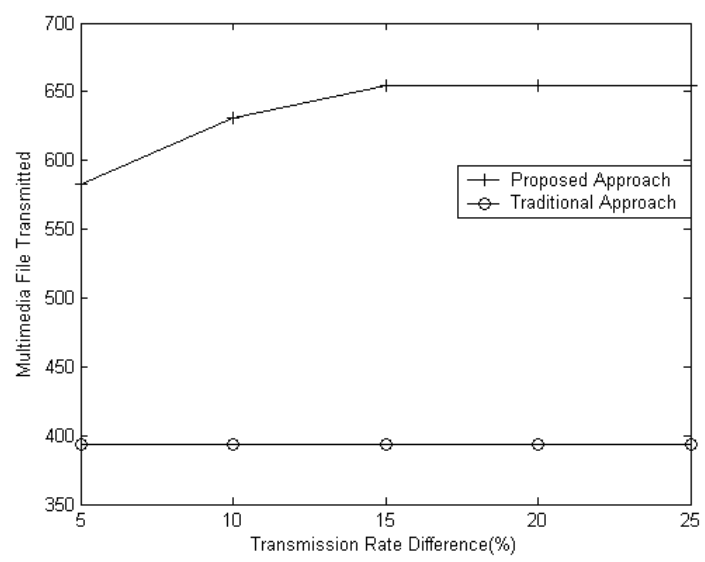

Figure 4: The number of passed file with various transmission rate differences (minimum end to end quality requirement is $356510659 \mathrm{MSE}$, bit loss provability $=3 / 100000)$

Figure 4 illustrates how many multimedia files could be transmitted before the first router runs out of the battery. The number of transmitted files can represent the lifetime of the wireless network directly, so it is used to estimate the 
performance of lifetime extension achieved by the proposed approach. From the figure we can see the proposed approach can transmits 50\% more multimedia files than traditional approach, which means the lifetime of the network is significantly extended by the new scheduling. The lifetime of the network depends on the router with the lowest energy, and the traditional approach always adds the maximum channel coding redundancy to the "lowest energy" router, so the lifetime of network does not change with various transmission rate differences. As Figure 3 shows, the energy-difference can not be totally balanced by the proposed approach if transmission rate difference is more than $15 \%$, and in this case, the proposed approach always allocate the minimum channel coding rate to the "lowest power" router to improve the energy-difference of the network if quality requirement is satisfied, so the number of transmitted multimedia files does not change after the transmission rate difference is greater than $15 \%$. However, if the transmission rate difference less than $15 \%$ and the energy-difference can be balanced by the proposed approach, various channel coding rate rather than always the minimum coding rate are applied to the "lowest energy" router to keep the energy-balancing, so more energy is consumed to increase the quality and the number of transmitted file is reduced.

\section{CONCLUSION}

In this paper we have proposed a new quality-assured energybalancing scheduling approach to prolong the multi-hop wireless mesh network lifetime by jointly considering quality requirement and energy-difference. In the proposed approach, the optimal channel coding rate strategy is performed to each hop to improve the energy-balancing while meeting the lowest multimedia service quality requirement. Simulation results have shown the proposed approach can significantly improve the energy-balancing between routers and can prolong the lifetime of the network at the cost of acceptable quality reduction.

\section{ACKNOWLEDGMENTS}

This research was supported in parts by Electrical Engineering and Computer Science departmental EE revolving fund at South Dakota State University, the National Natural Science Foundation of China No. 60902043, No. 61001083 and the Basic Research Foundation of Tsinghua National Laboratory for Information Science and Technology (TNList).

\section{REFERENCES}

[1] H. Fattah and C. Leung, "An overview of scheduling algorithms in wireless multimedia networks," IEEE Wireless Communications, pp. 76-83, Oct. 2002.

[2] N. Mastronarde, D. S. Turaga, M. Van Der Schaar, "Collaborative resource exchange for peer-to-peer video streaming over wireless mesh networks," IEEE J. Select. Areas Commun., vol. 25, pp. 108-118, Jan. 2007.

[3] B. Hamdaoui, P. Ramanathan, "Cross-layer optimized conditions for QoS support in multi-hop wireless networks with MIMO links," IEEE J. Select. Areas Commun., vol. 25, no. 4, pp. 667-677, May 2007.

[4] S. Kompella, S. Mao, Y. T. Hou, and H. D. Sherali, "Cross-layer optimized multipath routing for video communications in wireless networks," IEEE J. Select. Areas Commun., vol. 25, no. 4, pp. 831-840, May 2007.
[5] Y. Andreopoulos, N. Mastronarde, and M. v. d. Schaar, "Cross-Layer Optimized Video Streaming Over Wireless Multihop Mesh Networks," IEEE J. Select. Areas Commun., vol. 24, no. 11, pp. 2104-2115, Nov. 2006

[6] X. Tong, Y. Andreopoulos, and M. v. d. Schaar, "Distortion-driven video streaming over multihop wireless networks with path diversity," IEEE Trans. Mobile Computing, vol. 6, no. 12, pp. 1343-1356, Dec. 2007.

[7] L. Zhou, X. Wang, W. Tu, G.-M. Muntean, and B. Geller, "Distributed Scheduling Scheme for Video Streaming over Multi-Channel Multi-Radio Multi-Hop Wireless Networks," IEEE J. Select. Areas Commun., vol. 28, no. 3, pp. 409-419, Apr. 2010.

[8] H.-P. Shiang and M. v. d. Schaar, "Informationally Decentralized Video Streaming over Multi-hop Wireless Networks," IEEE Trans. Multimedia, vol. 9, no. 6, pp. 1299-1313, Sep 2007.

[9] K. T. Phan, H. Jiang, C. Tellambura, S. A. Vorobyov, and R. Fan, "Joint Medium Access Control, Routing and Energy Distribution in Multi-Hop Wireless Networks," IEEE Trans. Wireless Commun., vol. 7, no. 12, pp. 5244-5249, Dec. 2008.

[10] M. Cao, X. Wang, S.-J. Kim, and M. Madihian, "Multi-Hop Wireless Backhaul Networks: A Cross-Layer Design Paradigm," IEEE J. Select. Areas Commun., vol. 25, no. 4, pp. 738-748, May 2007.

[11] W. Wang, S. Shin, "A New Green Scheduling Approach to Maximize Wireless Multimedia Networking Lifetime via Packet and Path Diversity," in Proc. ACM Reliable and Autonomous Computational Science, Oct 2010.

[12] L. Xing, W. Wang, S. Wu, K. Hua, H. Wang "An Energy-Balanced Coding Redundancy Scheduling Approach to Support Quality of Service in Battery-Powered Multi-Hop Wireless Networks," in Proc. the 44th Annual Simulation Symposium (ANSS), Apr. 2011.

[13] B. Sklar, Digital Communications, $2^{\text {nd }}$ ed., Prentice Hall. 